\title{
A 3D Fractional-Order Chaotic System with Only One Stable Equilibrium and Controlling Chaos
}

\author{
Shiyun Shen, ${ }^{1}$ Meihua $\mathrm{Ke},{ }^{2}$ and Ping Zhou ${ }^{1,2}$ \\ ${ }^{1}$ Center of System Theory and Its Applications, Chongqing University of Posts and Telecommunications, Chongqing 400065, China \\ ${ }^{2}$ Key Laboratory of Network Control and Intelligent Instrument, Ministry of Education, \\ Chongqing University of Posts and Telecommunications, Chongqing 400065, China
}

Correspondence should be addressed to Ping Zhou; zhouping@cqupt.edu.cn

Received 5 March 2017; Accepted 5 June 2017; Published 5 July 2017

Academic Editor: Chris Goodrich

Copyright (C) 2017 Shiyun Shen et al. This is an open access article distributed under the Creative Commons Attribution License, which permits unrestricted use, distribution, and reproduction in any medium, provided the original work is properly cited.

\begin{abstract}
One 3D fractional-order chaotic system with only one locally asymptotically stable equilibrium is reported. To verify the chaoticity, the maximum Lyapunov exponent (MAXLE) with respect to the fractional-order and chaotic attractors are obtained by numerical calculation for this system. Furthermore, by linear scalar controller consisting of a single state variable, one control scheme for stabilization of the 3D fractional-order chaotic system is suggested. The numerical simulations show the feasibility of the control scheme.
\end{abstract}

\section{Introduction}

Fractional-order calculus is an old branch of mathematics, which can be dated back to the 17 th century $[1,2]$. Now, it is well-known that many real-world physical systems [1-4] can be more accurately described by fractional-order differential equations, for example, dielectric polarization, viscoelasticity, electrode-electrolyte polarization, electromagnetic waves, diffusion-wave, superdiffusion, heat conduction. Meanwhile, chaotic behavior has been found in many fractional-order systems like the fractional-order brushless DC motor chaotic system $[5,6]$, the fractional-order gyroscopes chaotic system [7], the fractional-order microelectromechanical chaotic system $[8]$, the fractional-order electronic circuits $[9,10]$, and so forth [11-16].

Recently, a simple three-dimensional autonomous chaotic system [17] with only one stable node-focus equilibrium has been reported by Wang and Chen. Due to the impossibility of existence of homoclinic orbit and the unique stable node-focus equilibrium in this striking chaotic system, the well-known Si'lnikov criterions are not applicable. To verify the chaoticity in this system, Wang and Chen [17] calculated the largest Lyapunov exponent, fractional dimension, and continuous broad frequency spectrum by numerical calculation. Huan et al. presented a rigorous computer-assisted verification of horseshoe chaos by virtue of topological horseshoe theory [18]. Up to now, some integer order chaotic systems with stable node-focus equilibrium have been presented. To the best of our knowledge, many previous fractional-order chaotic systems like the fractionalorder Lorenz chaotic system [19], the fractional-order Chen chaotic system [20], the fractional-order Lu chaotic system [21], the fractional-order brushless DC motor chaotic system $[5,6]$, the fractional-order gyroscopes chaotic system [7], the fractional-order microelectromechanical chaotic system [8], and so forth [9-14, 22, 23] have unstable equilibrium. There are seldom results on fractional-order chaotic systems with stable equilibrium. Hence, the finding of fractional-order chaotic systems with stable equilibrium is still an open problem.

Motivated by the above discussions, a three-dimensional autonomous fractional-order chaotic system with only one locally asymptotically stable equilibrium is proposed in this paper. The argument of all eigenvalues at equilibrium point satisfies $\left|\arg \left(\lambda_{i}\right)\right| \geq 0.5 \pi(i=1,2,3)$. Up to now, to the best of our knowledge, there are few results about the fractionalorder chaotic systems with stable equilibrium. To verify the chaoticity in this fractional-order system, the maximum 
Lyapunov exponent and chaotic attractors are yielded by numerical calculation. Furthermore, one control scheme for stabilization of this fractional-order chaotic system is suggested via linear scalar controller consisting of a single state variable. The numerical simulations show the feasibility of the control scheme. The organization of this paper is as follows: in Section 2, a new fractional-order chaotic system with only one stable equilibrium is presented, and the maximum Lyapunov exponent and chaotic attractors are obtained. In Section 3, in order to stabilize this fractional-order chaotic system, one control scheme is suggested via linear scalar controller consisting of a single state variable. The conclusion is finally drawn in Section 4.

\section{A New Fractional-Order Chaotic Systems with Only One Stable Equilibrium}

In this paper, the $q$-order Caputo derivative for function $y(t)$ is defined as follows:

$$
{ }_{0}^{C} D_{t}^{q} y(t)=\frac{1}{\Gamma(n-q)} \int_{0}^{t}\left[\frac{d^{n} y(\tau)}{d \tau^{n}}\right](t-\tau)^{n-q-1} d \tau,
$$

where $n-1 \leq q<n \in Z^{+}$and $\Gamma(n-q)=\int_{0}^{+\infty} t^{n-q-1} e^{-t} d t$ is the Gamma function.

Now, we consider the following 3D nonlinear fractionalorder system:

$$
\begin{aligned}
& { }_{0}^{C} D_{t}^{q} x_{1}(t)=\left(x_{2}(t)+\frac{1}{16}\right) x_{3}(t), \\
& { }_{0}^{C} D_{t}^{q} x_{2}(t)=x_{1}^{2}(t)+0.5 x_{1}(t)-x_{2}(t), \\
& { }_{0}^{C} D_{t}^{q} x_{3}(t)=-2 x_{1}(t)
\end{aligned}
$$

and, here, the fractional-order is $0<q<1$.

First, let us recall the stability theorem for nonlinear commensurate fractional-order systems.

Lemma 1 (see [24, 25]). Consider the following fractionalorder nonlinear system:

$$
{ }_{0}^{C} D_{t}^{q} x(t)=g(x(t))
$$

where $0<q<1$ is fractional-order, $x(t) \in R^{n}$ are state variables, and $g: R^{n} \rightarrow R^{n}$ is a nonlinear continuous vector function. The equilibrium point $\bar{x}$ (i.e., $g(\bar{x})=0$ ) in nonlinear fractional-order system (3) is locally asymptotically stable if $\left|\arg \left(\lambda_{i}(J)\right)\right|>0.5 \pi q(i=1,2, \ldots, n)$. Here, $J$ is the Jacobian matrix of $g(x(t))$ at equilibrium point $\bar{x}$ and $\lambda_{i}(i=1,2, \ldots, n)$ are the eigenvalues of matrix $J$.

Now, we can obtain that system (2) has only one equilibrium point; that is, $\left(\bar{x}_{1}, \bar{x}_{2}, \bar{x}_{3}\right)=(0,0,0)$. Meanwhile, we can yield the eigenvalues of Jacobian matrix at equilibrium point as follows: $\lambda_{1}=-1$, and $\lambda_{ \pm}= \pm 0.25 \sqrt{2} i$. So, we can obtain $\left|\arg \left(\lambda_{i}\right)\right| \geq 0.5 \pi>0.5 \pi q(i=1,2,3)$. According to the lemma, the equilibrium point $\left(\bar{x}_{1}, \bar{x}_{2}, \bar{x}_{3}\right)=(0,0,0)$ is locally asymptotically stable. Therefore, the equilibrium in system (2) is asymptotically stable.
Next, we discuss the numerical solution for system (2). Based on [20], we set $h=T / N$ and $t_{n}=n h(n=0,1$, $2, \ldots, N)$ and let initial condition be $\left(x_{1}(0), x_{2}(0), x_{3}(0)\right)$. So, the fractional-order system (2) can be discretized as follows:

$$
\begin{aligned}
& x_{1}(n+1)=x_{1}(0)+\frac{h^{q}}{\Gamma(q+2)}\left\{\left[x_{2}^{p}(n+1)+\frac{1}{16}\right]\right. \\
& \left.\cdot x_{3}^{p}(n+1)+\sum_{j=0}^{n} \alpha_{1, j, n+1}\left[x_{2}(j)+\frac{1}{16}\right] x_{3}(j)\right\}, \\
& x_{2}(n+1)=x_{2}(0)+\frac{h^{q}}{\Gamma(q+2)}\left\{\left[\left(x_{1}^{p}(n+1)\right)^{2}\right.\right. \\
& \left.+0.5 x_{1}^{p}(n+1)-x_{2}^{p}(n+1)\right] \\
& \left.+\sum_{j=0}^{n} \alpha_{2, j, n+1}\left[\left(x_{1}(j)\right)^{2}+0.5 x_{1}(j)-x_{2}(j)\right]\right\} \\
& \left.+\sum_{j=0}^{n} \alpha_{3, j, n+1}\left[-2 x_{1}(j)\right]\right\} \\
& x_{3}(n+1)=x_{3}(0)+\frac{h^{q}}{\Gamma(q+2)}\left\{\left[-2 x_{1}^{p}(n+1)\right]\right.
\end{aligned}
$$

Where

$$
\begin{aligned}
& x_{1}^{p}(n+1)=x_{1}(0)+\frac{1}{\Gamma(q)} \sum_{j=0}^{n} b_{1, j, n+1}\left[x_{2}(j)+\frac{1}{16}\right] x_{3}(j), \\
& x_{2}^{p}(n+1) \\
& =x_{2}(0)+\frac{1}{\Gamma(q)} \sum_{j=0}^{n} b_{2, j, n+1}\left[\left(x_{1}(j)\right)^{2}+0.5 x_{1}(j)-x_{2}(j)\right], \\
& x_{3}^{p}(n+1)=x_{3}(0)+\frac{1}{\Gamma(q)} \sum_{j=0}^{n} b_{3, j, n+1}\left[-2 x_{1}(j)\right],
\end{aligned}
$$$$
\alpha_{i, j, n+1}
$$$$
= \begin{cases}n^{q+1}-(n-q)(n+1)^{q}, & j=0, \\ (n-j+2)^{q+1}+(n-j)^{q+1}-2(n-j+1)^{q+1}, & 1 \leq j \leq n, \\ 1, & j=n+1,\end{cases}
$$$$
(i=1,2,3)
$$

$b_{i, j, n+1}=\frac{h^{q}}{q}\left[(n-j+1)^{q}-(n-j)^{q}\right], \quad 0 \leq j \leq n$.

The error of this approximation is described as

$$
\begin{aligned}
& \left|x_{i}\left(t_{n}\right)-x_{i}(n)\right|=o\left(h^{p}\right) \\
& \quad(i=1,2,3), p=\min (2,1+q) .
\end{aligned}
$$

Now, some results are obtained by numerical calculation. Let $q=0.96$; Figures $1(\mathrm{a})$ and $1(\mathrm{~b})$ show the results with initial condition $\left(x_{1}(0), x_{2}(0), x_{3}(0)\right)=(-1.2,1,1)$. 


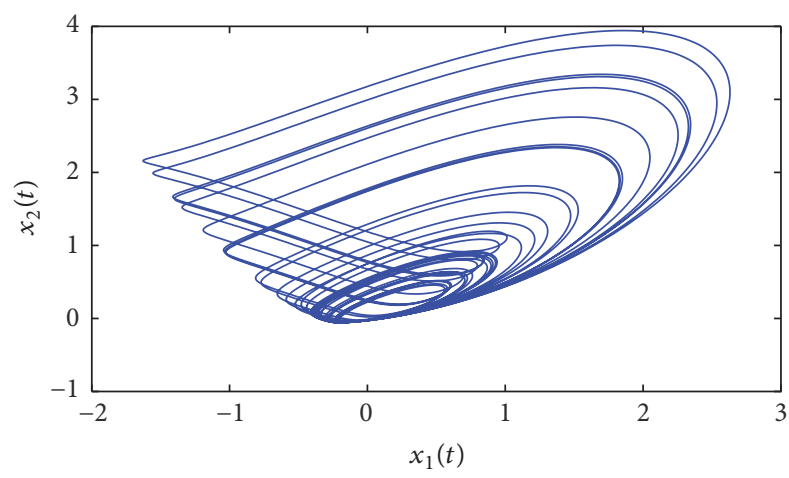

(a)

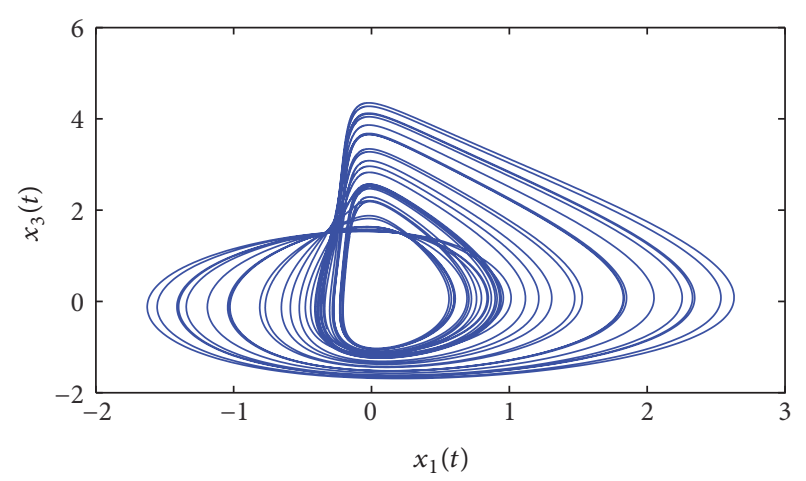

(b)

Figure 1: A chaotic attractor in system (2) for $q=0.96$.

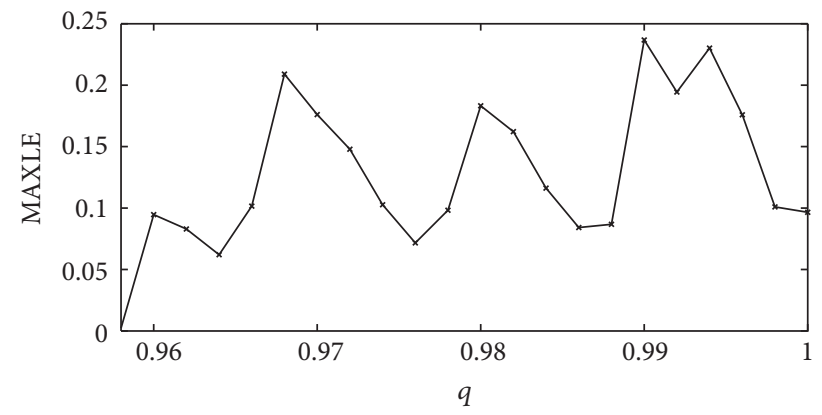

FIgURE 2: The maximum Lyapunov exponent (MAXLE) varies as fractional-order $q$.

The results in Figure 1 indicate that system (2) has a chaotic attractor if initial conditions are chosen as $\left(x_{1}(0), x_{2}(0), x_{3}(0)\right)=(-1.2,1,1)$.

To verify the chaoticity in system (2), we choose the initial conditions as $\left(x_{1}(0), x_{2}(0), x_{3}(0)\right)=(-1.2,1,1)$ and calculate the maximum Lyapunov exponent (MAXLE) of system (2) with respect to the fractional-order $q$ by numerical calculation. We obtain that the maximum Lyapunov exponent (MAXLE) is larger than zero for $0.958 \leq q \leq 1$. Figure 2 shows the maximum Lyapunov exponent (MAXLE) varies as fractional-order $q$. So, the chaotic attractor is emerged in system (2) for $0.958 \leq q \leq 1$.

For example, the MAXLE is 0.0022 when $q=0.958$, and its chaotic attractor is shown as Figures 3(a) and 3(b), while the MAXLE is 0.0946 when $q=0.96$, and its chaotic attractor is shown as Figure 1.

\section{Controlling Chaos via Linear Scalar Controller Consisting of a Single State Variable}

Now, we discuss how to stabilize the fractional-order chaotic system (2) via linear scalar controller consisting of a single state variable. We suggest the following controlled fractionalorder system:

$$
\begin{aligned}
& { }_{0}^{C} D_{t}^{q} x_{1}(t)=\left(x_{2}(t)+\frac{1}{16}\right) x_{3}(t)+K x_{2}(t), \\
& { }_{0}^{C} D_{t}^{q} x_{2}(t)=x_{1}^{2}(t)+0.5 x_{1}(t)-x_{2}(t), \\
& { }_{0}^{C} D_{t}^{q} x_{3}(t)=-2 x_{1}(t) .
\end{aligned}
$$

Here $K x_{2}(t)$ is the linear scalar controller consisting of a single state variable and $K<0$ is control parameter.

The controlled fractional-order system (7) is stable if the maximum Lyapunov exponent (MAXLE) of system (7) is negative. So, we can choose suitable control parameter $K$ such that the MAXLE of system (7) is negative. Now, we can calculate the MAXLE of system (7) by numerical calculation. The MAXLE of system (7) varying with respect to fractionalorder $q$ for control parameter $K$ is shown in Figure 4 , and here the initial condition is $\left(x_{1}(0), x_{2}(0), x_{3}(0)\right)=(-1.2,1,1)$.

The results in Figure 4 indicate that the controlled fractional-order system ( 7 ) is stable if $K=-1,-2,-3$, and -4 . It means that the fractional-order chaotic system (2) can achieve stability via scalar controller consisting of a single state variable $x_{2}(t)$.

For example, let $q=0.98$ and $K=-1$, and we can obtain MAXLE $=-0.2125$. So, the controlled fractional-order system (7) is stable. Figure 5 shows the simulative results. Let $q=0.96$ and $K=-2$, and we have MAXLE $=-0.1236$. So, the controlled fractional-order system (7) is stable. Figure 6 shows the simulative results. Let $q=0.97$ and $K=-3$, and we can obtain MAXLE $=-0.0774$. So, the controlled fractional-order system (7) is stable. Figure 7 shows the simulative results. Let $q=0.99$ and $K=-4$, and we have obtained MAXLE $=-0.0805$. So, the controlled fractionalorder system (7) is stable. Figure 8 shows the simulative results. Here, the initial condition is $\left(x_{1}(0), x_{2}(0), x_{3}(0)\right)=$ $(-1.2,1,1)$. 


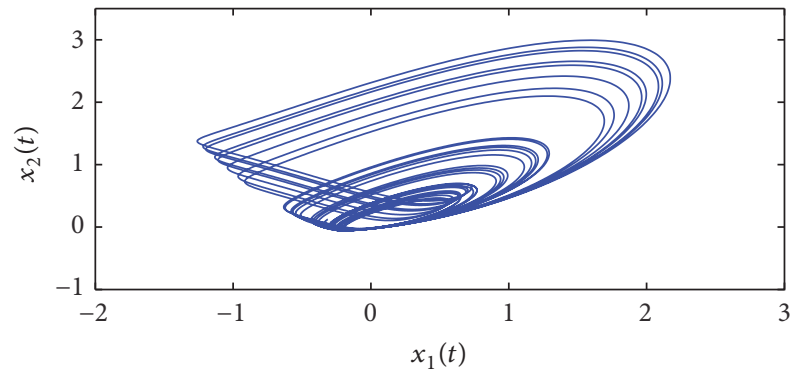

(a)

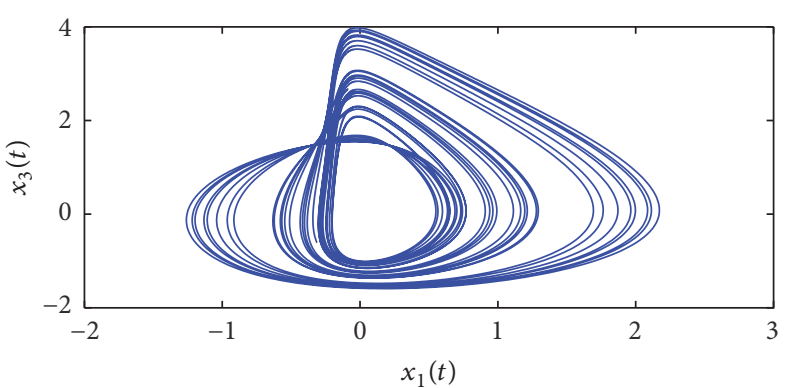

(b)

FIGURE 3: A chaotic attractor in system (2) for $q=0.958$.

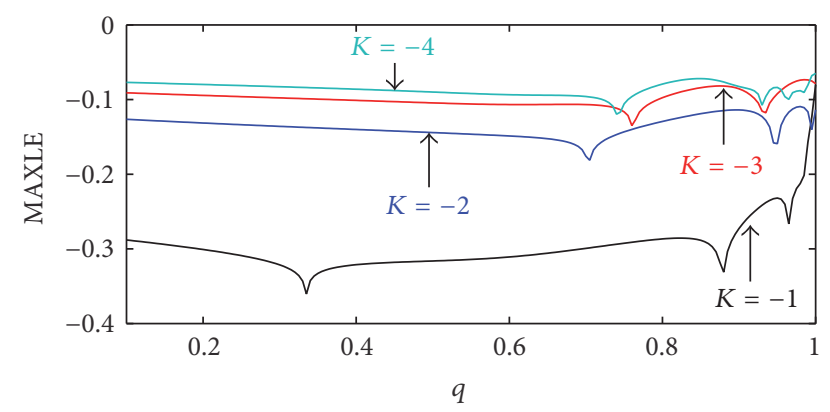

Figure 4: The MAXLE of system (7) varies as fractional-order $q$ for some control parameters $K$.

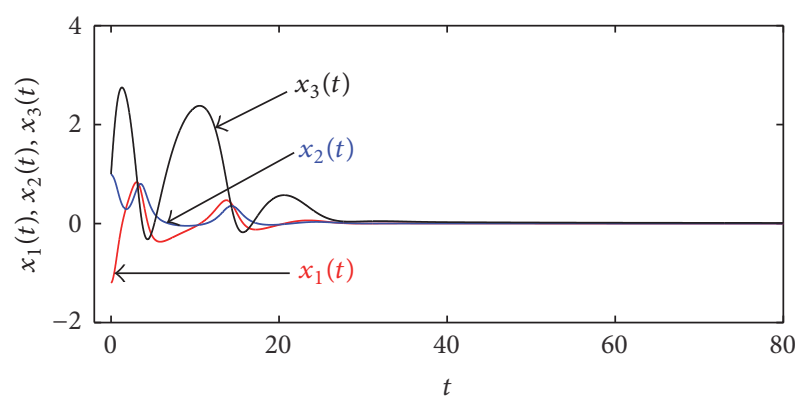

Figure 5: Stabilization of system (7) for $q=0.98$ and $K=-1$.

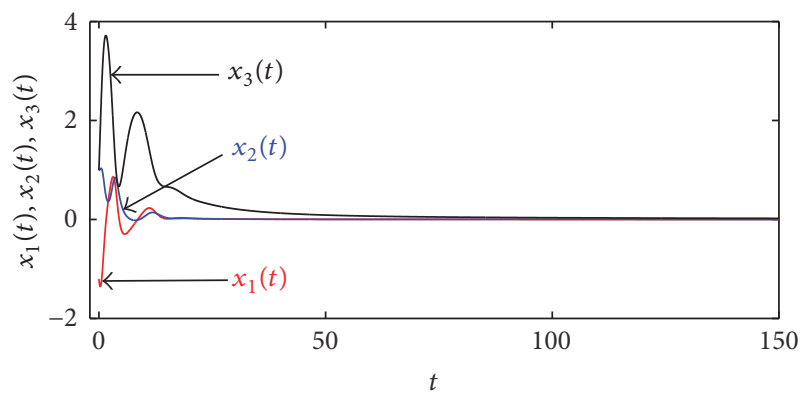

Figure 6: Stabilization of system (7) for $q=0.96$ and $K=-2$.

\section{Conclusions}

One new fractional-order chaotic system with only one stable equilibria point is reported in this paper. By numerical calculation, we yield the maximum Lyapunov exponent spectrum

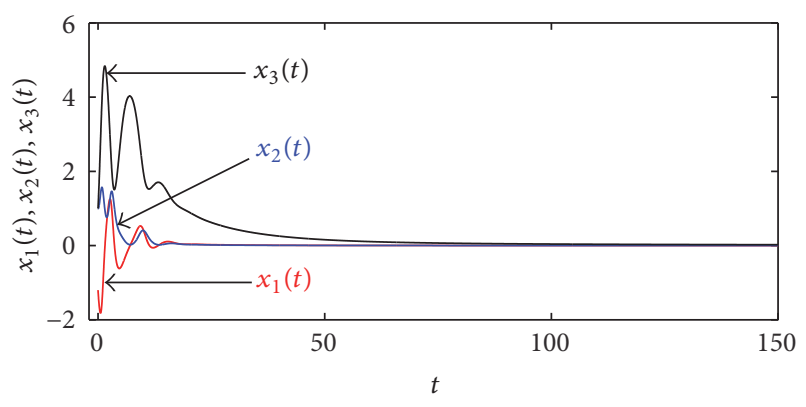

FIgURE 7: Stabilization of system (7) for $q=0.97$ and $K=-3$.

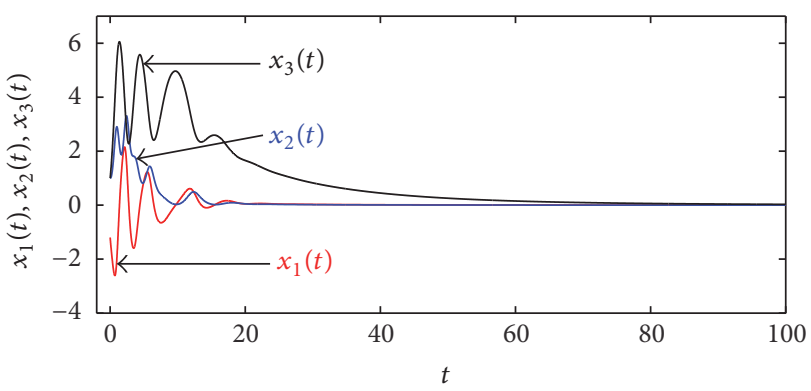

Figure 8: Stabilization of system (7) for $q=0.99$ and $K=-4$.

for this new fractional-order chaotic system, and the chaotic attractor can been found when $0.958 \leq q \leq 1$. The chaotic attractors for $q=0.958$ and $q=0.96$ are given. Meanwhile, in order to stabilize this fractional-order chaotic system, one control scheme is proposed via linear scalar controller consisting of a single state variable. By calculating the maximum Lyapunov exponent spectrum of the controlled system, we find that the maximum Lyapunov exponent (MAXLE) of system (7) is negative for suitable control parameter $K$. This result means that the new fractional-order chaotic system can achieve stability by the proposed control scheme. Some simulation results are given to show the validity and feasibility of our control scheme.

\section{Conflicts of Interest}

The authors declare no conflicts of interest. 


\section{Authors' Contributions}

All authors contributed equally to this work. All authors have read and approved the final manuscript.

\section{References}

[1] I. Podlubny, Fractional Differential Equations, Academic Press, San Diego, Calif, USA, 1999.

[2] R. Hilfer, Applications of Fractional Calculus in Physics, World Scientific, Singapore, 2000.

[3] L. P. Chen, Y. G. He, Y. Chai, and R. C. Wu, "New results on stability and stabilization of a class of nonlinear fractional-order systems," Nonlinear Dynamics, vol. 75, no. 4, pp. 633-641, 2014.

[4] C. P. Li, Z. G. Zhao, and Y. Q. Chen, "Numerical approximation of nonlinear fractional differential equations with subdiffusion and superdiffusion," Computers and Mathematics with Applications, vol. 62, no. 3, pp. 855-875, 2011.

[5] P. Zhou, H. Cai, and C. D. Yang, "Stabilization of the unstable equilibrium points of the fractional-order BLDCM chaotic system in the sense of Lyapunov by a single-state variable," Nonlinear Dynamics, vol. 84, no. 4, pp. 2357-2361, 2016.

[6] P. Zhou, R. G. Bai, and J. M. Zheng, "Stabilization of a fractionalorder chaotic brushless DC motor via a single input," Nonlinear Dynamics, vol. 82, no. 1, pp. 519-525, 2015.

[7] M. P. Aghababa and H. P. Aghababa, "The rich dynamics of fractional-order gyros applying a fractional controller," Proceedings of the Institution of Mechanical Engineers, Part I: Journal of Systems and Control Engineering, vol. 227, no. 7, pp. 588-601, 2013.

[8] M. P. Aghababa, "Chaos in a fractional-order micro-electromechanical resonator and its suppression," Chinese Physics $B$, vol. 21, no. 10, Article ID 100505, 9 pages, 2012.

[9] T. T. Hartley, C. F. Lorenzo, and H. K. Qammer, "Chaos in a fractional order Chua's system," IEEE Transactions on Circuits and Systems I: Fundamental Theory and Applications, vol. 42, no. 8, pp. 485-490, 1995.

[10] H. Y. Jia, Z. Q. Chen, and G. Y. Qi, "Chaotic characteristics analysis and circuit implementation for a fractional-order system," IEEE Transactions on Circuits and Systems I: Regular Papers, vol. 61, no. 3, pp. 845-853, 2014.

[11] P. Zhou, R. G. Bai, and J. M. Zheng, "Projective synchronization for a class of fractional-order chaotic systems with fractionalorder in the (1,2) interval," Entropy, vol. 17, no. 3, pp. 1123-1134, 2015.

[12] C. D. Yang, H. Cai, and P. Zhou, "Compound generalized function projective synchronization for fractional-order chaotic systems," Discrete Dynamics in Nature and Society, vol. 2016, Article ID 7563416, 8 pages, 2016.

[13] C. M. Jiang, S. T. Liu, and D. Wang, "Generalized combination complex synchronization for fractional-order chaotic complex systems," Entropy, vol. 17, no. 8, pp. 5199-5217, 2015.

[14] C. D. Yang, H. Cai, and P. Zhou, "Stabilization of the fractionalorder chua chaotic circuit via the caputo derivative of a single input," Discrete Dynamics in Nature and Society, vol. 2016, Article ID 4129756, 5 pages, 2016.

[15] J. Ma, X. Wu, R. Chu, and L. Zhang, "Selection of multi-scroll attractors in Jerk circuits and their verification using Pspice," Nonlinear Dynamics, vol. 76, no. 4, pp. 1951-1962, 2014.

[16] J. Ma, F. Q. Wu, G. D. Ren, and J. Tang, "A class of initialsdependent dynamical systems," Applied Mathematics and Computation, vol. 298, pp. 65-76, 2017.
[17] X. Wang and G. Chen, "A chaotic system with only one stable equilibrium," Communications in Nonlinear Science and Numerical Simulation, vol. 17, no. 3, pp. 1264-1272, 2012.

[18] S. Huan, Q. Li, and S. Yang, "Horseshoes in a chaotic system with only one stable equilibrium," International Journal of Bifurcation and Chaos in Applied Sciences and Engineering, vol. 23, no. 1, Article ID 1350002, 2013.

[19] I. Grigorenko and E. Grigorenko, "Chaotic dynamics of the fractional Lorenz system," Physical Review Letters, vol. 91, no. 3, pp. 034101-034104, 2003.

[20] C. P. Li and G. J. Peng, "Chaos in chen's system with a fractional order," Chaos, Solitons and Fractals, vol. 22, no. 2, pp. 443-450, 2004.

[21] W. H. Deng and C. P. Li, "Chaos synchronization of the fractional Lu system," Physica A: Statistical Mechanics and its Applications, vol. 353, no. 1-4, pp. 61-72, 2005.

[22] X. J. Wu, J. Li, and G. R. Chen, "Chaos in the fractional order unified system and its synchronization," Journal of the Franklin Institute, vol. 345, no. 4, pp. 392-401, 2008.

[23] J. Ma, F. Q. Wu, W. Y. Jin, P. Zhou, and T. Hayat, "Calculation of hamilton energy and control of dynamical systems with different types of attractors," Chaos: An Interdisciplinary Journal of Nonlinear Science, vol. 27, no. 5, Article ID 053108, 2017.

[24] K. Diethelm and N. J. Ford, "Analysis of fractional differential equations," Journal of Mathematical Analysis and Applications, vol. 265, no. 2, pp. 229-248, 2002.

[25] E. Ahmed, A. M. A. El-Sayed, and H.-A.-A. El-Saka, "Equilibrium points, stability and numerical solutions of fractionalorder predator-prey and rabies models," Journal of Mathematical Analysis and Applications, vol. 325, no. 1, pp. 542-553, 2007. 


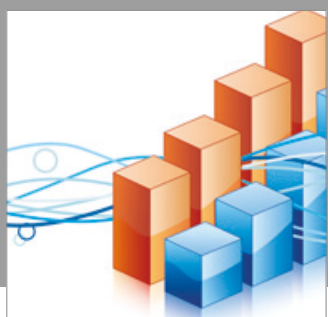

Advances in

Operations Research

vatersals

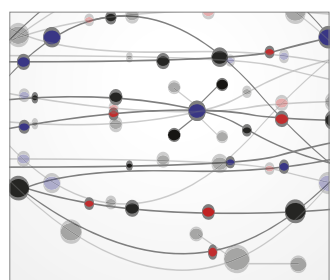

\section{The Scientific} World Journal
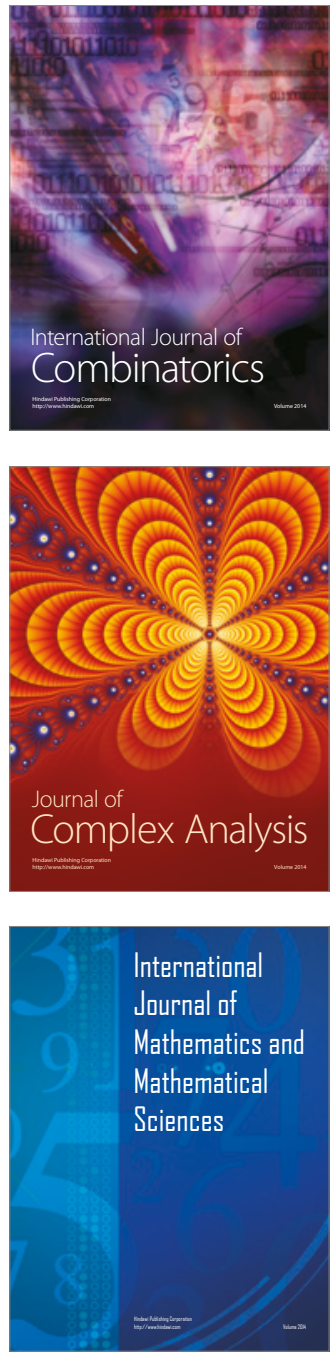
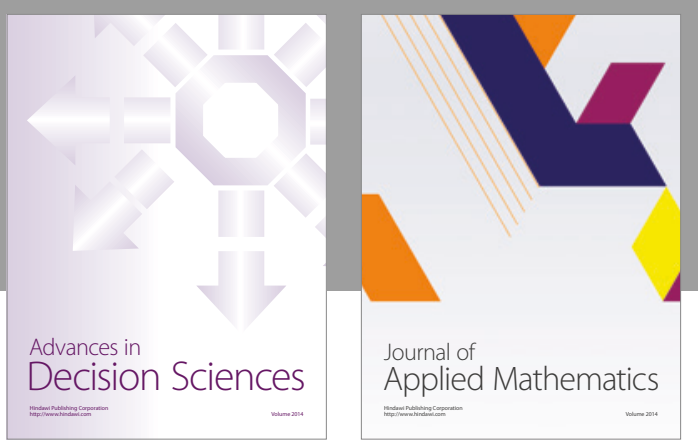

Algebra

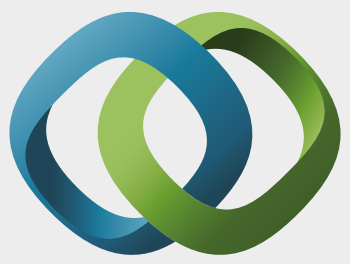

\section{Hindawi}

Submit your manuscripts at

https://www.hindawi.com
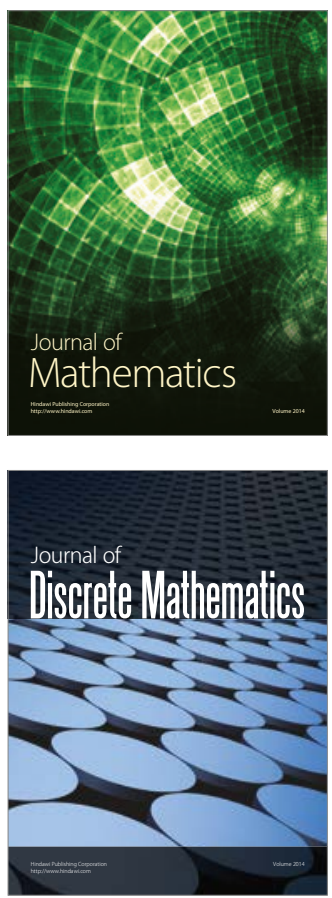

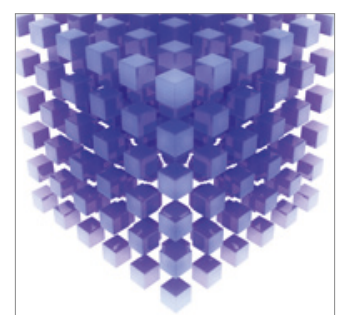

Mathematical Problems in Engineering
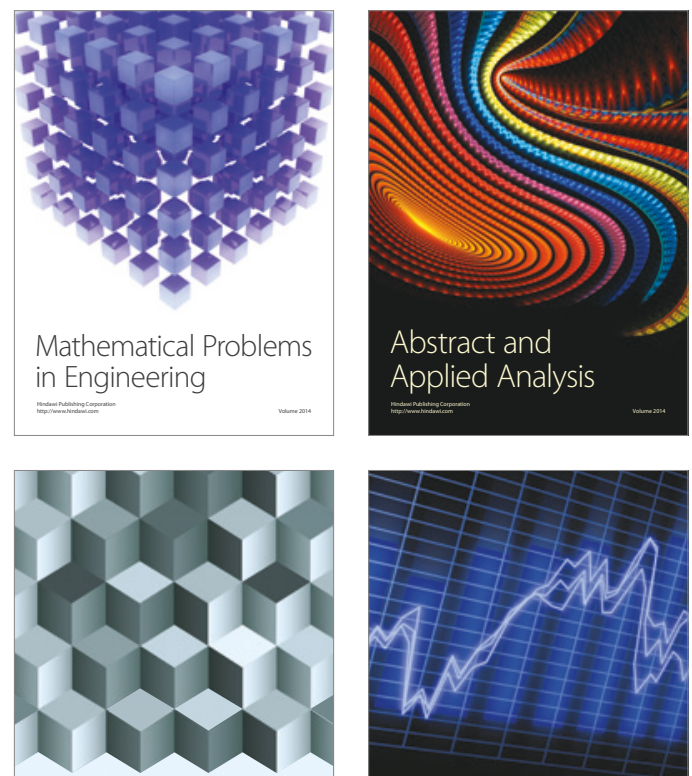

Journal of

Function Spaces

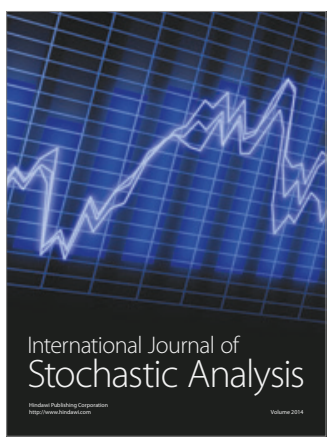

Probability and Statistics
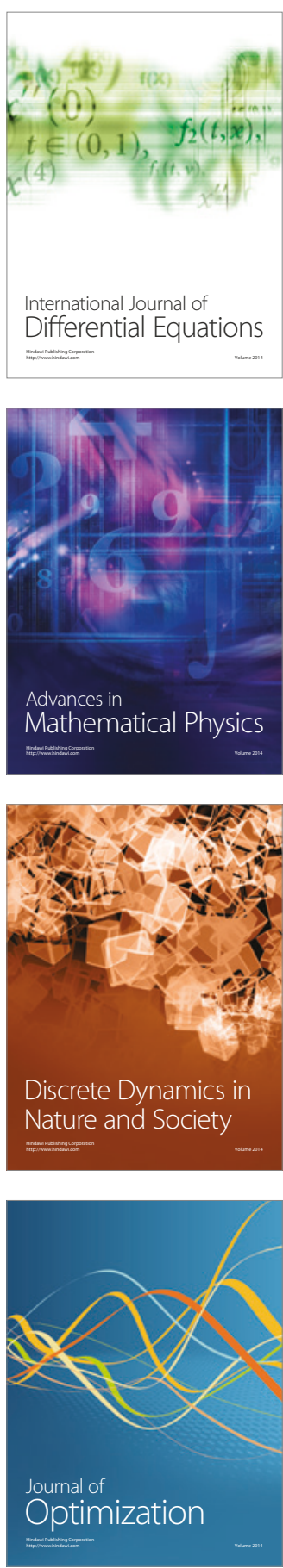